\begin{tabular}{ll}
\hline Breathlessness management & Physiotherapist \\
\hline Anxiety management part $1 \& 2$ & Complementary Therapist \\
Chair-based exercises & Physiotherapist/Occupational therapist \\
Energy conservation & Occupational Therapist \\
Tai Chi & Holistic Therapist \\
Pain management & Staff Nurse \\
Body image/self-esteem & Complementary Therapist \\
Nutrition & Staff Nurse \\
Creative therapy for well-being & Holistic Therapist \\
Reminiscence through music & Staff Nurse \\
\hline
\end{tabular}

provide opportunity for patients to have input with all members of the multi-disciplinary team. This helps patients to better understand their symptoms, empowering their use of resources and information to their advantage. Visual prompts include the use of an A1 flip chart and handouts to increase inclusion and underpin learning. The sessions provide peer support through informal discussions and can reassure patients that they are not alone; that they can talk openly, relate to each other and share experiences in a safe and reassuring environment.

Education Programme Evaluation The sessions support patients to reinforce existing coping strategies, develop and learn new ones. They aim to empower patients to alleviate and increase well-being. Importantly, the sessions prompt effective self-management of common symptoms experienced by palliative patients.

Conclusions The programme has helped to raise awareness of symptom control and has provided a holistic 'tool box' of self-help techniques to support patients in a personal sense of wellbeing and fulfilment; thus maximising enjoyment and quality of life.

\section{P-103 SELF-MANAGEMENT DVD: SUPPORT FOR PATIENTS IN THE HOME SETTING}

Jane Finnerty, Kelly De Souza, Tony Foster. Willowbrook Hospice, Prescot, UK

\subsection{6/bmjspcare-2016-001245.126}

Background Patients are living longer with a range of life limiting illnesses, the Wellbeing Service have adapted the Day Hospice education programme, and created with the assistance of a volunteer photographer a series of DVDs to help maximise patient's quality of life. Patients can access these free on discharge to enable on-going support at home.

Method The DVDs provide a visual and audio holistic 'tool box' of self-help techniques to reinforce self-management of common symptoms experienced by palliative patients. An audit from the education programme identified the following as key areas for support; breathlessness management, anxiety management, fatigue and mobility.

Topics on the DVDs include:

\begin{tabular}{ll}
\hline Self- massage/relaxed breathing techniques and guided & Complementary \\
visualisation & Therapist \\
Breathing techniques/relaxed positional techniques & Physiotherapist \\
Gentle chair based exercises & Occupational Therapist \\
Chair-based Tai Chi & Holistic Therapist \\
\hline
\end{tabular}

Results The DVDs may help patients to review and recap skills and coping strategies learnt within wellbeing services. They reinforce self-management of symptoms to support and empower patients with their own well-being thus maximising quality of life.

Benefits The DVDs:

- continue to provide reassurance and support at home following discharge

- may increase time between referrals back to service

- continue to empower and guide patients with their self-care

- may help carers to provide additional support to their loved ones' care, by increasing their knowledge and raising their awareness of strategies that patients can use to self-manage their symptoms

- provide a very good visual aid - especially to those that are hard of hearing. It may also be especially helpfully for patients that have short-term memory or impaired cognition.

Conclusions The DVDs provide on-going support post discharge for both the patient and their carers through the application of the techniques learnt at day hospice whilst in the comfort of their own home.

\section{P-104 SITTING DOWN BUT MOVING FORWARD!}

Jenny Sherburn. ellenor, Gravesend, UK

10.1136/bmjspcare-2016-001245.127

Background The opportunity to access exercise classes through the hospice is a more recent phenomenon, challenging the attitudes of staff and patients about the role of rehabilitation and exercise within palliative care. The physical and emotional benefits of exercise are well documented, so in response to this, and patient demand, 'drop in' seated exercise classes were created.

Aims

1. To provide the opportunity to exercise in a safe environment

2. To improve the fitness of the palliative patient group

3. To promote independence and wellbeing within the palliative patient group

4. To raise the profile of physiotherapy.

Approach A one-hour seated exercise session is delivered by a physiotherapist twice a week. Every attendee completes a Timed Up and Go test (T.U.G) prior to starting their first class, this test is repeated every fourth attendance.

Outcomes Classes have run for eight months, in that time 43 people attended. Out of these:

10 were both ambulatory and attended more than four sessions.

The T.U.G results are significant, demonstrating that $100 \%$ of participants achieved a quicker walk time on subsequent T.U.G testing.

Only one subject recorded a slower time on their fourth test. Conclusion These results suggest that participants have achieved an improvement in their fitness, and using the T.U.G as a guideline, $66 \%$ of attendees have reduced their walk time to $<14$ seconds and therefore, could have reduced their risk of falls.

The provision of a weekly exercise group has put physiotherapy 'on the map'. Verbal feedback from patients attending the group has been overwhelmingly positive: "It's made me feel more confident" and as a result the group is becoming more popular. 
These data shows that small service developments can make a big impact on palliative patients and the opportunity to exercise can and should be made available to all patients.

\section{P-105 ENGAGING PEOPLE TO EMPOWER PATIENTS}

${ }^{1,2}$ Karen Clarke. 'St Wilfrid's Hospice, Eastbourne, UK; ' Lancaster University, Lancaster, UK

\subsection{6/bmjspcare-2016-001245.128}

Background Despite having a life-limiting illness, patients wish to remain as independent as possible during their disease course (Yoshioka, 1994) and if this is optimised it can ameliorate their perceptions of hopelessness and despair and relieve some of the burden on the caregiver. Although intended to be supportive, evidence suggests that hospice palliative care may disable patients and reduce their ability to do things for themselves (Jennings, 2012).

Aims Explore how an approach to palliative care that focuses on maximising functional status, physical independence and quality of life, whilst acknowledging the patient's advanced disease and limited life span, can be integrated into a hospice in-patient setting.

Methods The setting is a 15 -bedded hospice inpatient unit.

Using participatory action research, a co-operative inquiry group (CIG) of clinical and non-clinical staff and volunteers collectively developed their knowledge in relation to a rehabilitative approach and organisational change; planned how to integrate a rehabilitative focus into the in-patient setting, attending to the potential facilitators and barriers; led on the agreed actions; met regularly to review progress and agree how the study should be evaluated.

Following the situational analysis, the CIG planned what action to take and after each period of activity, assessed the outcome and then further action was planned and executed.

Organisational change theory provides the theoretical lens for the study.

Results Achievements to date are aligned with Kotter's (2014) leading change model as follows:

- the CIG are excited and committed to change within the organisation, and

- have become the guiding coalition for the change process

- a vision is emerging to steer the change.

Conclusions Data collection will continue until June 2016 but early indications suggest that a group of hospice staff and volunteers identifying, owning and acting collaboratively, as described by Pascale and Sternin's (2005) positive deviance model, can form the basis of effective organisational change.

\section{P-106 THE POTENTIAL WITHIN US - SELF-MANAGEMENT THROUGH EXERCISE: A PHASED APPROACH TO RESEARCH}

\footnotetext{
${ }^{1}$ Abby McCarthy, ${ }^{2}$ Barbara Miller, ${ }^{2}$ Suzy Hudson, ${ }^{3}$ Sarah Klinger, ${ }^{4}$ Katie Margetts, 2,3 Karenann Spicer, ${ }^{5}$ Jo Clark, ${ }^{3}$ Trudy Leighton. ${ }^{1}$ Garden House Hospice, Letchworth, UK; ${ }^{2}$ The Hospice of St Francis; ${ }^{3}$ Peace Hospice Care; ${ }^{4}$ Isabel Hospice; ${ }^{5}$ Rennie Grove Hospice Care

10.1136/bmjspcare-2016-001245.129
}

Rehabilitative palliative care is a developing field integrating rehabilitation, enablement, self-management and self-care into the holistic model of palliative care. A growing evidence-base suggests that rehabilitation not only delays or prevents deterioration in function but can actively improve physical function irrespective of advance disease. The drive towards rehabilitative palliative care has led to an increase in the application of self-management techniques within hospices but there appears to be a gap in the empirical evidence specific to palliative care.

The challenges, possible solutions and anticipated patient benefits of hospices being research active have also been highlighted.

In response, palliative care professionals across five hospices formed a collaborative group and planned a phased approach to research.

Phase 1: A literature review (March - May 2016) to establish the evidence-base for self-management in palliative care. This identified a significant gap in evidencing the outcomes of selfmanagement in the palliative population.

Phase 2: A collaborative service evaluation (April - May, 2016) identifying self-management opportunities for patients within the hospices followed by a patient survey on the exercise groups they attended.

This suggested that promoting self-management through exercise was both acceptable and beneficial in a palliative population. Patient responses showed exercise:

- Enabled patients to cope better with their illness

- Increased their confidence in maintaining/improving activity levels

- Provided a focus for independent goal setting

- Facilitated independent activity outside of the organisation.

Phase 3: The work is being written up for publication to contribute to the evidence-base supporting the beneficial effects of self-management through exercise and the potential this has to improve patients' quality of life.

Phase 4: Moving forward the group aims to seek funding to extend this work into a research project and ultimately to drive further research through a dedicated research post.

\section{P-107 THE BE IN CHARGE PROGRAMME - SUPPORTS PATIENTS TO TAKE MORE CONTROL THEIR OWN CARE IN ORDER TO GET ON WITH THEIR LIFE}

Yvonne Cochrane, Denise Richards. Teesside Hospice, Middlesbrough, UK

\subsection{6/bmjspcare-2016-001245.130}

Background With the recognition of the growing number of people living longer with cancer and other life-limiting illness the hospice have undertaken a review of the service we offer.

Through a series of focus groups with patients and professionals from hospital and community teams it was evident that there was a need for a different kind of support for some patients. Therefore a new hospice service a six-week programme called the 'Be in Charge' programme was introduced.

Aim

- To help patients cope better with the symptoms of their illness and take more control in their own care

- To provide a space to talk and share experiences with other patients in similar situations

- To have an opportunity to learn coping skills to manage symptoms and the difficulties they may be experiencing

- The opportunity to have an introduction to the hospice and the services offered as well as identity a need for a different level of support 